\title{
Evaluating the predictive value of procalcitonin, cough duration, and heat peak in severe Mycoplasma pneumoniae pneumonia
}

\section{Ying Yang}

Women and Children's Hospital,School of Medicine,Xiamen university

\section{Liqing Chen}

Tongji Hospital of Tongji Medical College of Huazhong University of Science and Technology Yan Liu ( $\sim$ lyan3022@163.com )

Research article

Keywords: Mycoplasma pneumoniae pneumonia, procalcitonin, cough duration, heat peak

Posted Date: March 25th, 2020

DOl: https://doi.org/10.21203/rs.3.rs-19179/v1

License: (a) (i) This work is licensed under a Creative Commons Attribution 4.0 International License. Read Full License 


\section{Abstract}

Objective

To investigate the predictive value of risk factors for severe Mycoplasma pneumoniae pneumonia (MPP).

Methods

Retrospective analyses were conducted on 342 children with acute MPP hospitalized between December 2015 and December 2018.

Results

A total of 342 cases were including in the analyses, with most cases involving infants and preschoolaged children. Patients were categorized as general and severe MPP. Patients in the severe group had increased heat peak, fever duration, cough duration, length of hospitalization, and levels of white blood cell, neutrophils, C-reactive protein, and procalcitonin, as well as reduced levels of lymphocytes and platelets compared to the general group. Logistic regression and receiver operating characteristic curve analyses showed that increased levels of procalcitonin $(>0.67 \mathrm{ng} / \mathrm{mL})$, prolonged cough duration $(>13.5$ days), and heat peak above $38.4^{\circ} \mathrm{C}$ may be early predictors for severe MPP.

\section{Conclusions}

The severity of MPP effects fever, cough duration, and inflammatory response. Taking a detailed medical history and early procalcitonin measurement may help detect severe MPP.

\section{Background}

As the smallest prokaryote, Mycoplasma pneumoniae (MP) is a major pathogen of respiratory diseases in all age groups, but particularly in children and adolescents. The clinical manifestations of MP infections include self-limited upper respiratory tract infections and pneumonias, with the latter being responsible for $10-30 \%$ of all community-acquired pneumonias (CAPs) [1]. Macrolide-resistant MP has increased in the past 10 years, increasing by approximately $0-30 \%$ in Europe, 10\% in the United States and Canada, and 90\% in China and Japan [2,3]. Consequently, severe and refractory MPP cases have become increasingly common. While several studies have investigated risk factors for MPP and severe MPP, few have assessed their predictive value for severe MPP. In this study, the predictive value of risk factors for severe MPP were evaluated.

\section{Methods}

Study subjects 
Retrospective analyses were conducted on 342 children with MPP who were hospitalized between December 2015 to December 2018 in the pediatric departments of Tongji Hospital of Huazhong University of Science and Technology and Women and Children's Hospital of Xiamen University. All cases were confirmed according to the guidelines for CAP and MP infections [4-6]. Exclusion criteria included disease course > 1 month; mixed infections; congenital lung, chronic, or genetic metabolic disease; antibiotic treatment $>1$ week before admission; and insufficient data. After exclusions, a total of 342 children with MPP were included in the analyses. The cases were divided into two groups: general MPP $(n=275)$ and severe MPP $(n=67)$. Patients with severe MPP met at least one of the following criteria: severe toxicosis symptom or dyspnea; radiological deterioration observed in more than two lobes of the lung, pleural thickening, adhesion, pleural effusion, atelectasis, or pulmonary abscess; and severe extrapulmonary complications, severe organ damage, or severe disturbance of acid-alkaline balance and electrolyte [5,7-10]. Demographic and clinical data including age, sex, season of onset, heat peak(before admission), fever duration(before admission), cough duration(before admission), length of hospitalization (in days), and laboratory results were collected for all participants.

Detection of procalcitonin

Procalcitonin (PCT) levels were measured using electrochemiluminescence immunoassays after admission with a detection limit of $0.02 \mathrm{ng} / \mathrm{mL}$. The blood was drawn in vacuum tube filled with separation gel and centrifuged at $3500 \mathrm{rpm}$ for 5 minutes, and PCT levels were analyzed by Roche cobas 8000 automatic biochemistry analyzer within 30 minutes.

Statistical analyses

Categorial data are expressed as frequencies and the differences between the two groups were compared using chi-square and Fisher's exact tests. Continuous data are expressed as mean \pm standard deviation and the differences between the two groups were compared using independent samples t-tests. Multivariate logistic regression analyses and receiver operating characteristic (ROC) curves were used to assess the risk factors and their predictive value for severe MPP. All statistical analyses were performed using SPSS version 25.0 (IBM Corp, Armonk, NY, USA). P-values $<0.05$ were considered statistically significant.

\section{Results}

Population

Among the 342 patients with MPP, the average age was $4.07 \pm 2.57$ years old ( $54.4 \%$ male). Of all participants, $77.8 \%$ were infants or preschool-aged children. The incidence rate by season was $36.5 \%$ in spring, $28.4 \%$ in summer, $26.6 \%$ in autumn, and $8.5 \%$ in winter. A total of 208 cases $(60.8 \%)$ reported a fever.

Clinical characteristics and laboratory detection 
There were no differences in age, sex, season of onset, and prevalence of fever between the two groups. However, heat peak, fever duration, cough duration, and length of hospitalization were increased in patients with severe MPP compared to those with general MPP (Table 1). Moreover, patients with severe MPP had increased levels of white blood cell(WBC), neutrophils, C-reactive protein (CRP), and PCT, and reduced levels of lymphocytes and platelets (Table 2). 
Table 1

Demographic and clinical characteristics

\begin{tabular}{|c|c|c|c|c|}
\hline & $\begin{array}{l}\text { Severe MPP } \\
(n=67)\end{array}$ & $\begin{array}{l}\text { General MPP } \\
(n=275)\end{array}$ & $\mathrm{F} / \mathrm{X} 2$ & $P$ value \\
\hline Average age (years) & $3.77 \pm 2.57$ & $4.13 \pm 2.58$ & & 0.32 \\
\hline Sex & & & 0.16 & 0.69 \\
\hline Male & 35 & 151 & & \\
\hline Female & 32 & 124 & & \\
\hline Season of onset & & & 3.88 & 0.27 \\
\hline Spring & 22 & 103 & & \\
\hline Summer & 21 & 77 & & \\
\hline Fall & 15 & 76 & & \\
\hline Winter & 9 & 19 & & \\
\hline Fever & 49 & 159 & 3.37 & 0.07 \\
\hline Heat peak $\left({ }^{\circ} \mathrm{C}\right)$ & & & 47.54 & $\otimes 0.01 *$ \\
\hline Low fever(37.3-38.0) & 2 & 77 & & \\
\hline Moderate fever(38.1-39.0) & 22 & 60 & & \\
\hline High fever(39.1-41.0) & 24 & 20 & & \\
\hline Overhigh fever( $(\$ 41.0)$ & 1 & 2 & & \\
\hline Fever duration (days) & & & 28.90 & $\nabla 0.01^{*}$ \\
\hline$\otimes 7$ & 20 & 128 & & \\
\hline $7-13$ & 26 & 30 & & \\
\hline $14-20$ & 2 & 1 & & \\
\hline $21-30$ & 1 & 0 & & \\
\hline Cough duration (days) & & & 35.56 & $\otimes 0.01^{*}$ \\
\hline$\otimes 7$ & 3 & 26 & & \\
\hline $7-13$ & 22 & 109 & & \\
\hline $14-20$ & 28 & 37 & & \\
\hline
\end{tabular}




\begin{tabular}{|lllll|}
\hline & $\begin{array}{l}\text { Severe MPP } \\
(\mathbf{n}=67)\end{array}$ & $\begin{array}{l}\text { General MPP } \\
(\mathbf{n}=275)\end{array}$ & F/X2 & P value \\
\hline $21-30$ & 14 & 6 & 19.90 & $\varangle 0.01^{*}$ \\
\hline Hospitalization days & & & & \\
\hline$\square 7$ & 24 & 173 & \\
\hline $7-14$ & 40 & 101 & \\
\hline$\geq 14$ & 3 & 1 & \\
\hline * is Fisher test. & & & \\
\hline
\end{tabular}

Table 2

Laboratory detection

\begin{tabular}{|c|c|c|c|c|}
\hline & $\begin{array}{l}\text { Severe MPP } \\
(n=67)\end{array}$ & $\begin{array}{l}\text { General MPP } \\
(n=275)\end{array}$ & $\mathbf{t}$ & $P$ value \\
\hline $\operatorname{WBC}\left(\times 10^{9} / \mathrm{L}\right)$ & $10.45 \pm 6.92$ & $8.81 \pm 3.93$ & -2.07 & 0.04 \\
\hline Neutrophil rate(\%) & $53.86 \pm 19.47$ & $40.95 \pm 16.68$ & -5.68 & $\llbracket 0.01$ \\
\hline Lymphocyte rate(\%) & $34.40 \pm 18.19$ & $48.90 \pm 17.20$ & 6.09 & $\otimes 0.01$ \\
\hline Hemoglobin(g/L) & $117.50 \pm 10.42$ & $120.10 \pm 8.25$ & 1.98 & 0.05 \\
\hline Platelet $\left(\times 10^{9} / \mathrm{L}\right)$ & $318.10 \pm 98.81$ & $352.00 \pm 86.20$ & 2.87 & 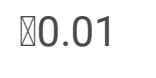 \\
\hline $\mathrm{CRP}(\mathrm{mg} / \mathrm{L})$ & $29.38 \pm 41.14$ & $10.78 \pm 10.55$ & -3.71 & $\llbracket 0.01$ \\
\hline PCT(ng/mL) & $2.55 \pm 5.49$ & $0.45 \pm 0.48$ & -3.05 & $\varangle 0.01$ \\
\hline
\end{tabular}

Logistic regression and ROC curve analyses

ROC curve analyses were conducted for PCT levels, cough duration, heat peak, and lymphocytes (Table 3). The analyses found that the optimal cutoff value for PCT was $0.67 \mathrm{ng} / \mathrm{mL}$, with an area under the curve (AUC) of 0.835 , sensitivity of $64.2 \%$, and specificity of $89.8 \%$. The optimal cutoff value for cough duration was 13.5 days, with an AUC of 0.805 , sensitivity of $62.7 \%$, and specificity of $85.5 \%$. The optimal cutoff value for heat peak was $38.4{ }^{\circ} \mathrm{C}$, with an AUC of 0.761 , sensitivity of $71.6 \%$, and specificity of $83.3 \%$ (Fig. 1). Lymphocytes were excluded from the analyses, as the ROC curve was below the diagonal line. 
Table 3

Multivariate logistic regression analyses

\begin{tabular}{|c|c|c|c|c|c|c|}
\hline \multirow[t]{2}{*}{ Variable } & \multirow[t]{2}{*}{ B } & \multirow[t]{2}{*}{ Wald } & \multirow[t]{2}{*}{$P$ value } & \multirow[t]{2}{*}{ OR } & \multicolumn{2}{|l|}{$95 \% \mathrm{Cl}$} \\
\hline & & & & & Lower limit & Upper limit \\
\hline Heat peak & 0.680 & 5.831 & 0.016 & 1.975 & 1.137 & 3.431 \\
\hline Fever duration & -0.035 & 0.201 & 0.654 & 0.965 & 0.827 & 1.127 \\
\hline Cough duration & 0.251 & 24.251 & 0.000 & 1.285 & 1.163 & 1.420 \\
\hline Hospitalization days & -0.078 & 0.584 & 0.445 & 0.925 & 0.758 & 1.129 \\
\hline WBC & -0.087 & 3.013 & 0.083 & 0.916 & 0.831 & 1.011 \\
\hline Neutrophil & -0.028 & 1.043 & 0.307 & 0.972 & 0.920 & 1.026 \\
\hline Lymphocyte & -0.068 & 5.432 & 0.020 & 0.934 & 0.882 & 0.989 \\
\hline Platelet & -0.003 & 1.382 & 0.240 & 0.997 & 0.993 & 1.002 \\
\hline CRP & 0.020 & 2.631 & 0.105 & 1.020 & 0.996 & 1.046 \\
\hline РСТ & 0.934 & 14.888 & 0.000 & 2.544 & 1.583 & 4.087 \\
\hline
\end{tabular}

\section{Discussion}

MP is an important cause of pediatric CAP and is estimated to be responsible for $10-40 \%$ of all CAP cases [3]. In China, there are up to 2.7 million pediatric severe CAP every year [6], with $13.0-42.6 \%$ of cases being severe MPP [11]. This study assessed the clinical characteristics of pediatric patients with acute MPP to evaluate the risk factors and their predictive values for severe MPP. To date, there is limited evidence on the age, sex, and seasonal characteristics of MPP. A 10-year longitudinal study in North China found that the peak age of MPP was 6-10 years old, accounting for $75.2 \%$ of all cases in children [11]. However, in our study, the majority of patients with MPP were infants or preschool-aged children.

This finding is consistent with a study based in Thailand [12]. Regional differences in the season of onset have been demonstrated, with increased incidence in the fall and winter in North China, and increased incidence in the summer and fall in South China [11]. Moreover, previous studies have reported an association between MPP and elevated atmospheric temperatures and humidity, with a $17 \%$ increase in MPP cases for every $1{ }^{\circ} \mathrm{C}$ increase, and a $4 \%$ increase in MPP cases for every $1 \%$ humidity increase [6, 13]. In the present study, patients lived in Central and South China, and most MPP cases occurred in the spring, summer, and fall, similar to previous studies [6, 13]. Nevertheless, no significant differences in age, sex, and seasonal onset between patients with general MPP and severe MPP were observed in this study. Further large-scale, multicenter studies are needed to validate these findings. 
The most common symptoms of MPP were fever and cough. Fever was reported in $73.1 \%$ of patient with severe MPP and $57.8 \%$ of patients with general MPP. While not statistically significant, fevers tended to be higher in patients with severe MPP. Most children with severe MPP had moderate or high fevers, whereas most children with general MPP had moderate or low fever. The majority of the patients with severe MPP had a fever lasting more than 7 days, a cough lasting more than 2 weeks, and most required hospitalization for more than 7 days. Patients with general MPP had significantly shorter fever durations, cough durations, and hospitalizations compared to those with severe MPP, consistent with previous reports $[8,14,15]$. The Japanese Respiratory Society's scoring system provides guidance for recognizing MPP, with white blood cell (WBC) count $<10 \times 10^{9} / \mathrm{L}$ being one of six standard parameters [3]. In our study, patients with MPP had WBC counts below this threshold. Patients with severe MPP had significantly increased levels of neutrophils, CRP and PCT, as well as reduced levels of lymphocytes and platelets compared to patients with general MPP. These findings are consistent Yan et al. [8, 16, 17]. Previous studies have suggested that thrombocytopenia is an extrapulmonary manifestation of MPP [3, 18]. Therefore, acute-phase thrombocytopenia may have predictive value for severe MPP. In general, the presence of pyrogenic products of inflammation and excessive inflammatory response can cause lung damage or even lead to necrotizing pneumonia [19-22].

In recent years, several studies have investigated the risk factors for MPP, but few have assessed their predictive value. Our ROC curve analyses showed that increased PCT levels $(>0.67 \mathrm{ng} / \mathrm{mL})$, prolonged cough duration (> 13.5 days), and heat peak above $38.4{ }^{\circ} \mathrm{C}$ may be early predictors for severe MPP in children. The analyses revealed that PCT offers slightly greater predictive value than cough duration and heat peak. Therefore, a detailed history of cough duration and heat peak may be required when assessing the risk for severe MPP [17]. In addition, PCT had greater specificity compared to the other two risk factors. PCT is a 116 amino acid protein encoded by the CALC-1 gene, which typically exists in low concentrations in parafollicular cells of the thyroid gland in healthy individuals. In the presence of an infection, CALC-1 gene expression is induced in non-neuroendocrine cells, resulting in increased PCT levels [23]. As a clinical biomarker of bacterial infections, PCT levels are measured to assess the severity and prognosis of CAP, acute respiratory distress syndrome, and sepsis, and guide antibiotic and glucocorticoid treatment [23-27]. While several studies have investigated the relationship between PCT and Streptococcus pneumoniae, Haemophilus influenzae, and other common bacterial pathogens in patients with CAP, few clinical studies have assessed this relationship in patients with MPP [28]. PCT levels increase within 2-4 h after infection and peak within 24-48 $\mathrm{h}$ [29]. The PCT assessment has several advantages including its availability, ease of use, low cost, universality, and reliability. Moreover, in this study, the threshold value for PCT was $0.67 \mathrm{ng} / \mathrm{mL}$, which is much lower than the threshold for severe bacterial infections and sepsis ( $\geq 2 \mathrm{ng} / \mathrm{mL}$ ). These findings are consistent with those of previous study that suggested that PCT levels may be used to predict severe MPP in patients with acute MPP [16]. In addition, PCT levels at admission were associated with longer fever duration and hospitalization, indicating that PCT may be a risk factor for prolonged fever in MPP [30].

\section{Conclusion}


This study found increased intrapulmonary and extrapulmonary complications as well as worse outcomes in patients with severe MPP compared to those with MPP. Moreover, the severity of disease was closely linked to clinical manifestations and the inflammatory response. Our findings suggest that in children with MPP, increased levels of procalcitonin $(>0.67 \mathrm{ng} / \mathrm{mL})$, prolonged cough duration $(>13.5$ days), and heat peak above $38.4{ }^{\circ} \mathrm{C}$ can be used as a reference index for the assessment of disease severity.

\section{Limitations}

There were some limitations in this study. First, it was a retrospective observational study and the enrolled patients were only from two tertiary centers. Second,some patients received antibiotic treatment before admission and it might affect the results of heat peak】cough duration and PCT levels. However, our evaluation had been done in a large study population even excluded the patients who received more than a week of antibiotic treatment and those with mixed infections. Furthermorelcases received antibiotic were the true status for most hospitalized MPP children. Therefore $\llbracket$ further prospective and multicenter studies are necessary to predict risk factors for severe MPP.

\section{Abbreviations}

MP

Mycoplasma pneumoniae

MPP

Mycoplasma pneumoniae pneumonia

CAPs

community-acquired pneumonia

WBC

white blood cell

CRP

C-reactive protein

PCT

procalcitonin

ROC

receiver operating characteristic

AUC

area under the curve

\section{Declarations}

\section{Ethical declarations}


This study was approved by the Ethics Committee of Tongji Hospital and all participants provided written informed consent from parents/guardians.

\section{Consent for publication}

Not applicable.

\section{Availability of data and materials}

All data generated or analyzed during the current study are available from the corresponding author Yan Liu on reasonable request. Data are not publicly available due to concerns regarding research participant privacy.

\section{Competing interests}

The authors declare no conflicts of interest relevant to this study.

\section{Funding}

Not applicable.

\section{Authors'contributions}

Liqing Chen and Ying Yang contributed to designing the study and interpreting data. Ying Yang contributed to drafting the manuscript. Yan Liu contributed to designing the study, acquisition of and interpreting data and approving the final version of the manuscript. All authors read and approved the final manuscript.

\section{Acknowledgements}

We are grateful to all patients participating in the trials discussed.

\section{References}

1. Qu J,Chen S,Bao F,et al.Molecular characterization and analysis of Mycoplasma pneumoniae among patients of all ages with community-acquired pneumonia during an epidemic in China. Int $\mathrm{J}$ Infect Dis.2019;83:26-31.

2. Wagner K, Imkamp F, Pires VP, et al. Evaluation of Lightmix Mycoplasma macrolide assay for detection of macrolide-resistant Mycoplasma pneumoniae in pneumonia patients. Clin Microbiol Infect.2019;25(3):383.e5-383.e7.

3. Bajantri B, Venkatram S, Diaz-Fuentes G, et al. Mycoplasma pneumoniae: A Potentially Severe Infection. J Clin Med Res.2018;10(7):535-544.

4. Michael $\mathrm{H}$,Julia $\mathrm{C}$,et al. British Thoracic Society guidelines for the management of community acquired pneumonia in children: update 2011. Thorax.2011;66(2):1-23. 
5. The Subspecialty Group of Respiratory Disease, The Society of Pediatrics, ChineseMedical Association, The Editorial Board, Chinese Journal of Pediatrics. Guidelinesfor management of community acquired pneumonia in children (the revised editionof 2013) (I). Chin J Pediatr. 2013;51:745-799.

6. Daxboeck F,Krause R,Wenisch C. Laboratory diagnosis of Mycoplasma pneumoniaeinfection. Clin Microbiol Infect.2003;9(4):263-273.

7. Bradley JS, Byington CL, Shah SS, et al. The Management of Community-AcquiredPneumonia in Infants and Children Older Than 3 Months of Age: Clinical PracticeGuidelines by the Pediatric Infectious Diseases Society and the Infectious DiseasesSociety of America. Clin Infect Dis. 2011;53(7):617-630.

8. Yan C, Xue G, et al. Molecular and clinical characteristics of severe Mycoplasma pneumoniae pneumonia in children. Pediatr Pulmonol; 2019;54(7):1012-1021.

9. Søndergaard MJ, Friis MB, et al. Clinical manifestations in infants and children with Mycoplasma pneumoniae infection. PLoS One. 2018;13(4):e0195288.

10. S.Valade, L.Biard, et al. Severe atypical pneumonia in critically ill patients: a retrospective multicenter study. Intensive Care. 2018;8:81.

11. Li-Wei Gao,Ju Yin,Ying-hui Hu,et al. The epidemiology of paediatric Mycoplasma pneumoniae pneumonia in North China: 2006 to 2016. Epidemiology and Infection.2019;147, e192:1-5.

12. Phares $C R$, Wangroongsarb $P$, Chantra $S$,et al. Epidemiology of severe pneumonia caused by Legionella longbeachae, Mycoplasmapneumoniae,and Chlamydia pneumoniae: 1year, population-based surveillance for severepneumonia in Thailand. Clin Infect Dis. 2007;45(12):147-155.

13. Onozuka D, Hashizume M, Hagihara A,et al. Impact of weather factors on Mycoplasma pneum-oniae pneumonia. Thorax.2009;64(6):507-511.

14. Le Wang, Zhishan Feng, et al. $\mathrm{QRisk}$ factors of 90-day rehospitalization followin discharge of pediatric patients hospitalized with mycoplasma Pneumoniae pneumonia. BMC Infectious Diseases.2019; 19(1) : 966.

15. Yuanyuan Zhang, Yunlian Zhou, et al. The Clinical Characteristics and Predictors of Refractory Mycoplasma pneumoniae Pneumonia in Children. PLoS One.2016;11(5):1-10.

16. Xiao-Hua Han, Li-Yun Liu, et al.Changes of inflammation-associated factors in children with Mycoplasma pneomoniaepneumonia and concomitant systemic inflammatory response syndrome.Zhongguo Dang Dai Er Ke Za Zhi.2007;9(4):347-350.

17. Xu JJ, Shu LH. Clinical characteristics of refractory Mycoplasma pneumoniae pneumonia in children. Zhongguo Dang Dai Er Ke Za Zhi. 2018;20(1):37-42. 
18. Narita M. Pathogenesis of extrapulmonary manifestations of Mycoplasma pneumoniae infection with special reference to pneumonia. J Infect Chemother. 2010;16(3):162-169.

19. Saraya T, Watanabe T, Tsukahara Y,et al. The correlation between chest $X$-ray scores and the clinical findings in children and adults with Mycoplasma pneumoniae pneumonia. Intern Med.2017;56(21): 2845-2849.

20. Jin-Rong Liu, Jie Lu, Fang Dong, et al. Low Bacterial Co-infection Invalidates the Early Use of Nonanti-Mycoplasma pneumonia Antibiotics in PediatricRefractory Mycoplasma pneumonia Pneumonia Patients.Front Pediatr.2018;26(6):296. 21.

21. Wang $X$, Zhong LJ, Chen ZM, et al. Necrotizing pneumonia caused by refractory Mycoplasma pneumonia pneumonia in children.World J Pediatr.2018;14(4):344-349.

22. Seo YH,Kim JS,Seo SC,et al. Predictive value of C-reactive protein in response to macroli-des in children with macrolide-resistant Mycoplasma pneumoniae pneumonial.Korean J Pediatr;2014, 57(4) ه86-92.

23. Sarah Chalmers,Ali Khawaja, et al.Diagnosis and treatment of acute pulmonary inflammation in critically ill patients:The role of inflammatory biomarkers. World J Crit Care Medl.2019;8(5):59-71.

24. Angela Branche, Olivia Neeser, et al. Procalcitonin to guide antibiotic decision making. Curr Opin Infect Dis.2019;32 (2):130-135.

25. Bachar Hamade, David T Huang, et al. Procalcitonin Where Are We Now?. Crit Care Clin.2020;36 (1):23-40.

26. Benita Tujula, Sari Hämäläinen, et al. Review of Clinical Practice Guidelines on the Use of Procalcitonin in Infections. Infect Dis (Lond).2019;Dec 20:1-8.

27. Akihiro Ito, Tadashi Ishida, et al. Serial Procalcitonin Levels for Predicting Prognosis in CommunityAcquired Pneumonia. Respirology.2016;21 (8):1459-1464.

28. The Special Committee of Hospital Infection Management and Control,the Society of Pediatrics, Chinese Medical Association, Chinese Journal of Pediatrics. Expert consensus on clinical application of serum procalcitonin in pediatric infectious disease. Chin J Pediatr.2019;57 (1) :9-15.

29. Meisner M. Update on procalcitonin measurements. Ann Lab Med.2014;34(4):263-273.

30. Jeong JE, Soh JE, Kwak JH, et al.Increased procalcitonin level is a risk factor for prolonged fever in children with Mycoplasmapneumonia.Korean J Pediatr. 2018;61(8):258-263.

\section{Figures}




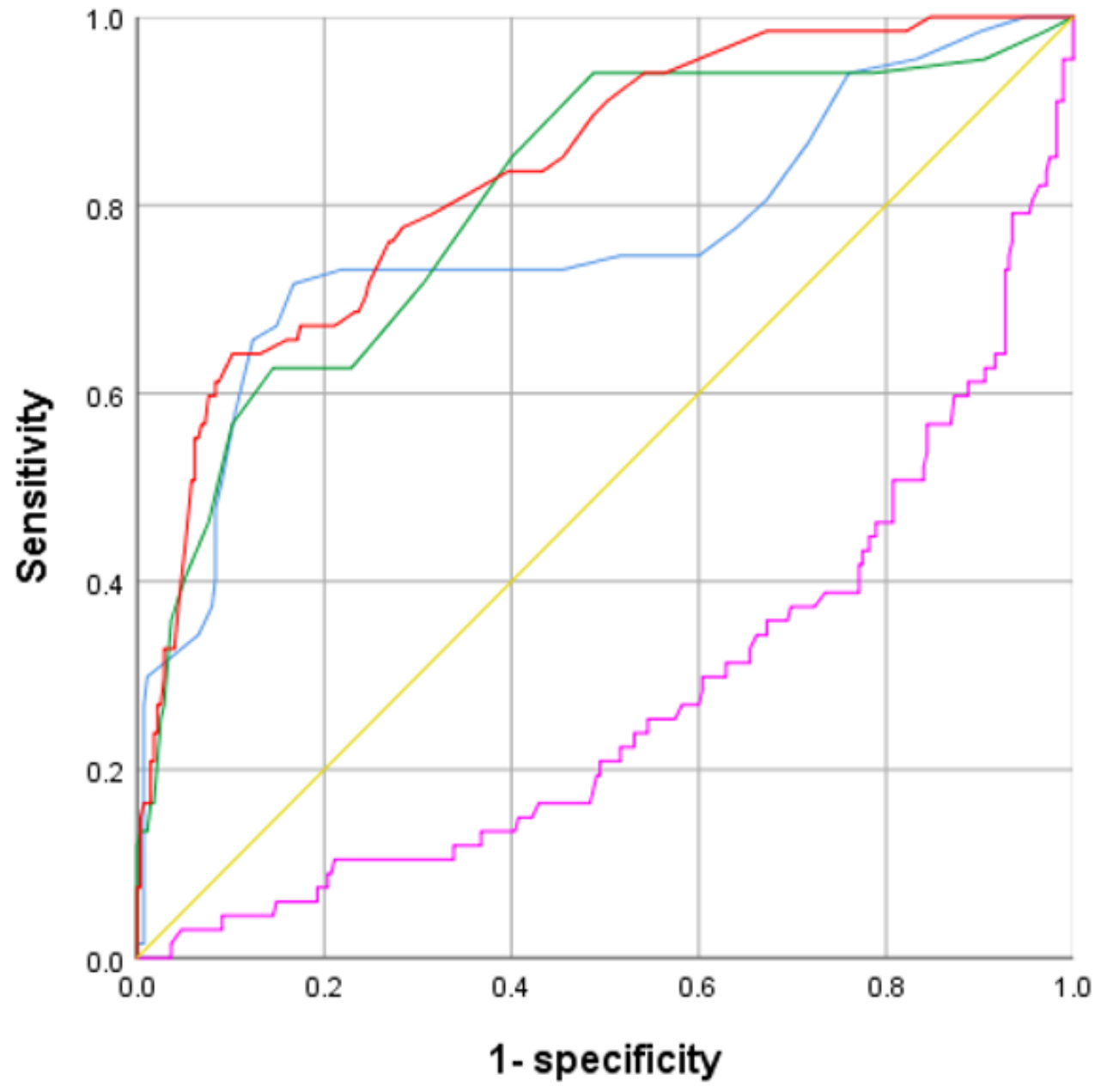

Source of the curve

Heat peak

Cough duration

Lymphocytes

PCT

Reference line

Figure 1

ROC curve of risk factors 